\title{
Bone mesenchymal stem cells co-expressing VEGF and BMP-6 genes to combat avascular necrosis of the femoral head
}

\author{
HONGXING LIAO ${ }^{1,2}$, ZHIXIONG ZHONG ${ }^{3}$, ZHANLIANG LIU ${ }^{1}$, \\ LIANGPING $\mathrm{LI}^{2}$, ZEMIN LING ${ }^{2}$ and XUENONG ZOU ${ }^{2}$ \\ ${ }^{1}$ Department of Orthopedics, Meizhou People's Hospital, Meizhou, \\ Guangdong 514000; ${ }^{2}$ Guangdong Provincial Key Laboratory of Orthopedics and Traumatology, \\ The First Affiliated Hospital of Sun Yat-sen University, Guangzhou, Guangdong 510080; ${ }^{3}$ Department of \\ Cardiovascular Medicine, Meizhou People's Hospital, Meizhou, Guangdong 514000, P.R. China
}

Received July 21, 2017; Accepted November 3, 2017

DOI: 10.3892/etm.2017.5455

\begin{abstract}
The aim of the present study was to investigate the potential of bone mesenchymal stem cells (BMSCs) treated with a combination of vascular endothelial growth factor (VEGF) and bone morphogenetic protein-6 (BMP-6) genes for the treatment of avascular necrosis of the femoral head (ANFH). Rat BMSCs were isolated and purified using a density gradient centrifugation method. The purity and characteristics of the BMSCs were detected by cell surface antigens identification using flow cytometry. The experimental groups were administered with one of the following adeno-associated virus (AAV) vector constructs: AAV-green fluorescent protein (AAV-GFP), AAV-BMP-6, AAV-VEGF or AAV-VEGF-BMP-6. The expression of VEGF and BMP-6 was detected by reverse transcription-quantitative polymerase chain reaction, western blotting and ELISA assays. The effects of VEGF and BMP-6 on BMSCs were evaluated by angiogenic and osteogenic assays. The transfected BMSCs were combined with a biomimetic synthetic scaffold poly lactide-co-glycolide (PLAGA) and they were then subcutaneously implanted into nude mice. After four weeks, the implants were analyzed with histology and subsequent immunostaining to evaluate the effects of BMSCs on blood vessel and bone formation in vivo. In the AAV-VEGF-BMP-6 group, the expression levels of VEGF and BMP-6 were significantly increased and human umbilical vein
\end{abstract}

Correspondence to: Dr Xuenong Zou, Guangdong Provincial Key Laboratory of Orthopedics and Traumatology, The First Affiliated Hospital of Sun Yat-sen University, 58 Zhongshan Er Road, Guangzhou, Guangdong 510080, P.R. China

E-mail: zxnong@hotmail.com

Dr Zhixiong Zhong, Department of Cardiovascular Medicine, Meizhou People's Hospital, 63 Huangtang Road, Meizhou, Guangdong 514000, P.R. China

E-mail:mzdrzhi@163.com

Key words: vascular endothelial growth factor, bone morphogenetic protein- 6 , bone mesenchymal stem cells, avascular necrosis of the femoral head endothelial cells tube formation was significantly enhanced compared with other groups. Capillaries and bone formation in the AAV-VEGF-BMP-6 group was significantly higher compared with the other groups. The results of the present study suggest that BMSCs expressing both VEGF and BMP-6 induce an increase in blood vessels and bone formation, which provides theoretical support for ANFH gene therapy.

\section{Introduction}

In China, the number of patients with avascular necrosis of the femoral head (ANFH) is estimated to be between 5 and 7 million and there are 100,000-150,000 new cases each year (1). At present, gene therapy is a large area of ANFH research (2). Basic fibroblast growth factor (bFGF), transforming growth factor- $\beta$ (TGF- $\beta$ ), bone morphogenetic proteins (BMPs), and vascular endothelial growth factor (VEGF) all serve important roles in the development of applicable gene therapies, in particular BMPs and VEGF (3-5). Bone morphogenetic proteins (BMPs) are signal molecules that promote bone regeneration (6-8). As important members of the TGF- $\beta$ superfamily, BMPs have effects on the stimulation of osteoblast differentiation $(9,10)$ and this process involves the differentiation of progenitor cells into chondrocytes and osteoblasts, endochondral ossification and new bone formation (11). Studies have revealed that BMP-2, BMP-4, BMP-6, BMP-7 and BMP-9 are crucial for bone formation (12-15). Furthermore, a number of studies have suggested that BMP-6 is the most potent osteoinductive BMP and has greater potential for bone regeneration than BMP-2 and BMP-4 (16-18). Angiogenesis is the most basic physiological process in bone repair (19); furthermore, VEGF, which is expressed by endothelial cells, is one of the most important cytokines in angiogenesis (20) and is associated with all steps of bone formation, including mesenchymal condensation, cartilage formation, cartilage resorption and blood vessel invasion $(21,22)$. It has been indicated that BMPs stimulate angiogenesis via the osteoblast production of VEGF-A, which plays an important role in bone formation and angiogenesis by acting as a chemoattractant (23). A number of studies have attempted to determine the effects of combining VEGF with BMP in order to encourage bone formation. One such study reported that 
the combination of VEGF and BMP-4 enhances bone formation $(24,25)$. Another revealed that, when combined, VEGF and BMP-2 are effective in stimulating bone regeneration (26). In addition, the co-administration of VEGF and BMP-4 has been reported to induce osteogenesis more effectively than VEGF and BMP-2 (27). These studies have focused on the delivery of BMP-2 and BMP-4 in conjunction with VEGF. However, to the best of our knowledge, few studies have focused on the effects of the combination of VEGF and BMP-6 on bone repair.

In the present study, adeno-associated viruses (AAV) co-expressing VEGF and BMP-6 were constructed to investigate the potential of bone mesenchymal stem cells (BMSCs) treated with a combination of VEGF and BMP-6 genes for the treatment of ANFH.

\section{Materials and methods}

Ethics statement. The animal study was approved by the First Affiliated Hospital of Sun Yat-sen University (Guangzhou, China).

Isolation, culture, and verification of rat BMSCs. A total of 20 healthy 3-4 weeks specific pathogen free grade Sprague Dawley rats (weighing 100-120 g, 10 males and 10 females) were purchased from the Animal Center of Sun Yat-Sen University (Guangzhou, China). The rats were housed in an environment of $25 \pm 5^{\circ} \mathrm{C}$ with $35 \pm 5 \%$ humidity and a standard $12 \mathrm{~h}$ light/dark cycle and ad libitum access to food and water. After three days, the rats were sacrificed by intraperitoneal injection of $300 \mathrm{mg} / \mathrm{kg}$ pentobarbital, soaked in $75 \%$ ethanol for $10 \mathrm{~min}$ and then the tibiae and femora were isolated under sterile condition. BMSCs were obtained from the tibiae and femora of the rats following sacrifice. Density gradient centrifugation and adherent screening were used to isolate BMSCs as previously described (28). In short, tibiae and femora were flushed with low glucose Dulbecco's modified Eagle's medium (L-DMEM, GE Healthcare Life Sciences, Logan, UT, USA) to harvest the BMSCs, which were cultured in L-DMEM supplemented with $10 \%$ fetal bovine serum (FBS, Gibco; Thermo Fisher Scientific, Inc., Waltham, MA, USA) and $20 \mathrm{mg} / \mathrm{ml}$ penicillin-streptomycin (Gibco; Thermo Fisher Scientific, Inc.). Cells were subsequently incubated with $5 \% \mathrm{CO}_{2}$ at $37^{\circ} \mathrm{C}$ for 7 days and split, following which cells at the fourth passage were used for the following experiments. The obtained cells were added into Percoll separation solution (Sigma Aldrich: Merck KGaA, Darmstadt, Germany) with a density of $1.073 \mathrm{~g} / \mathrm{ml}$ and centrifuged at $4,000 \mathrm{x}$ g for $25 \mathrm{~min}$ at $20^{\circ} \mathrm{C}$. The cells were further resuspended in complete medium containing $90 \%$ L-DMEM, $10 \% \mathrm{FBS}, 100 \mu \mathrm{l} / \mathrm{ml}$ penicillin and $100 \mu \mathrm{l} / \mathrm{ml}$ streptomycin (Gibco; Thermo Fisher Scientific, Inc.) and incubated at $37^{\circ} \mathrm{C}$ in a $5 \% \mathrm{CO}_{2}$ supplemented incubator (Thermo Fisher Scientific. Company, USA). Cell surface antigens were used to identify BMSC characteristics via flow cytometry. Briefly, cells were collected and washed twice with PBS (Gibco; Thermo Fisher Scientific, Inc.) and then incubated with $0.5 \%$ BSA blocking buffer in 1x PBS (Thermo Fisher Scientific, Inc.) for $30 \mathrm{~min}$ at $4^{\circ} \mathrm{C}$. Following washing with PBS, cells were immunofluorescently stained with fluorochrome-conjugated antibodies specific to the cell surface antigens cluster of differentiation CD90 (cat no. ab225; 1:200, Abcam, Cambridge, UK), CD29 (cat no. bs-20631R; 1:200; Bioss, Beijing, China), CD44 (cat no. bs-0521R; 1:200, Bioss), CD11 (cat no. bs-2508R; 1:200; Bioss), CD45 (cat no. bs-0522R; 1:200; Bioss) and CD34 (cat no. ab81289; $1: 200$, Abcam) at $4^{\circ} \mathrm{C}$ for $30 \mathrm{~min}$. Flow cytometry was performed on a FACSCanto II flow cytometer (BD Biosciences, Franklin Lakes, NJ, USA) using FACSDiva software (BD Biosciences) and the data was analyzed by FlowJo software version 10.06 (Tree Star, Inc., Ashland, OR, USA). Each surface antigen assay was performed in triplicate.

Adeno-associated virus type 2 (AAV) vector production and infection in vitro. The recombinant vector was packaged by the adeno-associated virus (AAV) Helper-Free System (Hanbio Biotechnology Co., Ltd, Shanghai, China). The internal ribosome entry site (IRES) fragment was incorporated into the plasmid AAV multiple cloning site (pAAV MCS) (Hanbio Biotechnology Co., Ltd) and sub-cloned into two multiple cloning sites (Hanbio Biotechnology Co., Ltd). Next, VEGF and BMP-6 primers were designed by Primer Premier 5 software (Premier Biosoft Inc., Palo Alto, CA, USA) and synthesized by Sangon Biotech Company (Shanghai, China), then VEGF and BMP-6 were inserted into the upstream and downstream MCS, respectively. The bicistronic frame was $2.5 \mathrm{~kb}$ in length, which is within the vector capacity. The recombinant (r)AAV-VEGF-IRES-BMP-6 (AAV-VEGF-BMP-6), rAAV-VEGF-green fluorescent protein (GFP) (AAV-VEGF), rAAV-BMP-6-GFP (AAV-BMP-6), and rAAV-IRES-GFP (AAV-GFP) vectors were co-transfected into AAV-293 cells (Forevergen, Guangzhou, China) with the plasmid (p)AAV-helper and pAAV-RC (carrying AAV-2 replication and capsid genes) using the calcium phosphate method by adding $\mathrm{CaCl}_{2}$, according to the manufacturer's instructions (Invitrogen; Thermo Fisher Scientific, Inc.). A primary virus stock was collected $72 \mathrm{~h}$ following transfection and chloroform/PEG8000 protocols as previously described (29) were used to concentrate and purify the primary virus stock. In order to achieve the greatest cytopathogenic effect, infection efficiency and cost of the r virus, it was determined that $5 \times 10^{4}$ viral particle/cell was the best multiplication of infection (MOI) for infecting rat BMSCs (30). The BMSCs $\left(2 \times 10^{5} / \mathrm{ml}\right)$ were seeded onto 96 -well plates and incubated for $30 \mathrm{~min}$ at $37^{\circ} \mathrm{C}$ in an atmosphere containing $5 \%$ $\mathrm{CO}_{2}$. BMSCs were subsequently transfected with AAV-GFP, AAV-VEGF, AAV-BMP-6, or AAV-VEGF-BMP-6 vectors at the optimum MOI using lipofectamine 2000 (Invitrogen; Thermo Fisher Scientific, Inc.). After 48 h, samples were collected for subsequent experimentation.

Reverse transcription-quantitative polymerase chain reaction $(R T-q P C R)$. Total RNA from BMSCs infected with the AAV-GFP, AAV-VEGF, AAV-BMP-6 or AAV-VEGF-BMP-6 viruses was extracted using TRIzol Reagent (Invitrogen; Thermo Fisher Scientific, Inc.) according to the manufacturer's protocol and reverse transcribed to cDNA with Super Script TM III Reverse Transcriptase (Invitrogen; Thermo Fisher Scientific, Inc.). The target genes were analyzed using a Takara SYBR $^{\circledR}$ Green I qPCR kit (Takara Bio, Inc. Otsu, Japan) performed on the ABI 7500 system (Applied Biosystems; Thermo Fisher Scientific, Inc.) using the following thermocycling conditions: $94^{\circ} \mathrm{C}$ for $3 \mathrm{~min}, 35$ cycles at $94^{\circ} \mathrm{C}$ for $30 \mathrm{sec}$, $55^{\circ} \mathrm{C}$ for $30 \mathrm{sec}$, and $72^{\circ} \mathrm{C}$ for $45 \mathrm{sec}$. The primers used were as follows: GAPDH, forward 5'-CCTCGTCTCATAGACAAG 
ATGGT-3' and reverse 5'-GGGTAGAGTCATACTGGAACA TG-3'; BMP6, forward 5'-ACAGCATAACATGGGGCTTC-3' and reverse 5'-CTCGGGGTTCATAAGGTGAA-3'; VEGF, forward 5'-TTGCTGCTCTACCTCCAC-3' and reverse 5'-AAT GCTTTCTCCGCTCTG-3'; osteocalcin (OCN), forward 5'-CTCTGTCTCTCTGACCTCACAG-3' and reverse 5'-GGA GCTGCTGTGACATCCATAC-3'; runt-related transcription factor 2 (RUNX2), forward 5'-GAAACTCTTGCCTCGTCC GCT-3' and reverse 5'-GATGATGACACTGCCACCTCTG-3'; alkaline phosphatase (AKP), forward 5'-CTGGTGGAAGGA GGCAGAATT-3' and reverse 5'-ATGTGAAGACGTGGG AATGGT-3'. The relative mRNA expression was calculated using the $2^{-\triangle \Delta C q}$ method (31).

ELISA. A total of $500 \mu 1$ from each culture supernatant was harvested from the AAV-GFP, AAV-VEGF, AAV-BMP-6 and AAV-VEGF-BMP- 6 transfection groups. VEGF and BMP-6 levels in the culture medium were examined using ELISA kits (Rat VEGF ELISA kit, cat no. RRV00, R\&D Systems, Minneapolis, MN, USA; and Rat BMP-6 ELISA kit, cat no. MBS704699, MyBiosource, San Diego, CA, USA) following manufacturer's protocol. The absorbance was determined at $450 \mathrm{~nm}$ using a microplate reader (Multiskan Go; Thermo Fisher Scientific, Inc.). The concentrations of VEGF and BMP- 6 were determined by comparing the absorbance with those of the standards.

Western blotting. For protein extraction, cells were lysed in cell lysis buffer containing $140 \mathrm{mM} \mathrm{NaCl}, 10 \mathrm{mM}$ Tris- $\mathrm{HCl}, 1 \%$ Triton X-100, 1 mM ECTA and 1X protease inhibitor cocktail (Gibco; Thermo Fisher Scientific, Inc.). The protein concentration was determined using the BCA assay. A total of $20 \mu \mathrm{g}$ protein was loaded per lane and separated on 12\% SDS-PAGE gels. Proteins were subsequently transferred to polyvinylidene difluoride membranes, which were blocked with $5 \%$ non-fat dry milk for $1 \mathrm{~h}$ at room temperature and incubated overnight at $4^{\circ} \mathrm{C}$ with the following primary antibodies: Anti-GAPDH (cat no. sc-25778; 1:2,000) anti-BMP6 (cat no. sc-7406; 1:100; both Santa Cruz Biotechnology, Inc.) and anti-VEGF (cat no. ab105219; 1:1,000; Abcam). They were subsequently incubated with an anti-goat horseradish peroxidase (HRP)-conjugated secondary antibody (cat no. sc-2004, 1:1,000, Santa Cruz Biotechnology, Inc.) at $4^{\circ} \mathrm{C}$ for overnight, and detected with an enhanced chemiluminescence detection kit (EMD Millipore, Billerica, MA, USA).

Angiogenic assay in vitro. The basement membrane Matrigel matrix (BD Biosciences,) was incubated at $37^{\circ} \mathrm{C}$ for $30 \mathrm{~min}$ in a 24-well plate following dilution with serum-free medium. When solidification occurred, human umbilical vein epithelial cells (HUVECs; $5 \times 10^{4}$ cells/well; (Forevergen) were seeded with fresh L-DMEM medium supplemented with $10 \%$ FBS. Tube formation of HUVECs was observed by co-culture with BMSCs. At 14 days following after infection, $1 \mathrm{ml}$ of each culture supernatant obtained from AAV-GFP, AAV-VEGF, AAV-BMP-6 and AAV-VEGF-BMP- 6 cells was added to the 24-well plate and incubated at $37^{\circ} \mathrm{C}$ for $12 \mathrm{~h}$ in an atmosphere containing $5 \% \mathrm{CO}_{2}$. Images of tube formation were acquired from three random fields under a light microscope (magnification, x200) and analyzed with Image Pro Plus 6.0 (Media Cybernetics, Inc., Rockville, MD, USA)
Osteogenic assay in vitro. Following transfection with AAV-GFP, AAV-VEGF, AAV-BMP-6 or AAV-VEGF-BMP-6, BMSCs were cultured in L-DMEM supplemented with $10 \%$ FBS containing $20 \mathrm{mg} / \mathrm{ml}$ penicillin-streptomycin in an atmosphere containing $5 \% \mathrm{CO}_{2}$ at $37^{\circ} \mathrm{C}$ for $30 \mathrm{~min}$. During the second week, the mineralization effects were detected by Von Kossa staining, alizarin red staining (ARS), and AKP staining. For AKP staining, cells were washed with PBS, fixed with $4 \%$ paraformaldehyde at $4^{\circ} \mathrm{C}$ overnight and stained with AKP solution at room temperature for 10 min (Beyotime Institute of Biotechnology). Images of mineral nodules were captured from three random fields under a light microscope (magnification, x200) and subsequently analyzed with Image Pro Plus 6.0 (Media Cybernetics, Inc.). For ARS staining, ARS was prepared in double distilled $\mathrm{H}_{2} \mathrm{O}$ and the $\mathrm{pH}$ was adjusted to 4.1 using $10 \%$ (v/v) ammonium hydroxide. Sections were fixed in $70 \%$ ethanol at room temperature for $10 \mathrm{~min}$ and then stained with $40 \mathrm{mM}$ ARS for $5 \mathrm{~min}$ at room temperature. Calcium deposition was assessed by eluting ARS staining with distilled water including 10\% acetic acid and $20 \%$ methanol. The absorbance of supernatants was measured at $405 \mathrm{~nm}$.

In vitro cell seeding on poly lactide-co-glycolide (PLAGA) scaffolds. The biomimetic synthetic PLAGA scaffolds were sterilized using $70 \%$ ethanol for $1 \mathrm{~h}$ and rinsed three times in PBS. PLAGA scaffolds were then exposed to ultraviolet light for $24 \mathrm{~h}$ and treated with PC-2000 plasma cleaner (South Bay Technology, Inc., San Clemente, CA, USA) to enhance cell attachment. Finally, the cells $\left(1.67 \times 10^{5} / \mathrm{ml}\right)$ were seeded on each PLAGA scaffold. A total of 126 weeks old nude mice (weighing 22-25 g; 6 males and 6 females) were purchased from the Animal Center of Sun Yat-sen University (Guangzhou, China). The rats were housed in an environment of $25 \pm 5^{\circ} \mathrm{C}$ with $35 \pm 5 \%$ humidity and a standard $12 \mathrm{~h}$ light/dark cycle and ad libitum access to food and water. After one day, the PLAGA scaffolds were implanted subcutaneously into the nude mice ( $\mathrm{n}=3$ per group). Implants were retrieved following 2 or 3 weeks, embedded in paraffin, sectioned into $5 \mathrm{~mm}$ slices and mounted onto glass slides.

Histological analysis of in vivo bone formation. Slides were washed in xylene twice for $10 \mathrm{~min}$ each in order to remove the paraffin. A graded series of ethanol solutions, including 100,95 , and $70 \%$ ethanol were used for rehydration. The sections were washed twice in deionized water for $5 \mathrm{~min}$ each. Tissues were fixed in $4 \%$ paraformaldehyde at $4^{\circ} \mathrm{C}$ for overnight, embedded in paraffin, sliced into sections $4 \mathrm{~mm}$ thick and stained with hematoxylin and eosin (H\&E) at room temperature for $15 \mathrm{~min}$. For Von Kossa staining, sections were stained with von Kossa stain at room temperature for 20 min to examine calcium deposition. In addition, immunohistochemistry was used to examine the expression of VEGF and BMP-6 in implants. Fixed tissues were deparaffinized, incubated in citrate buffer at room temperature for $10 \mathrm{~min}$ and subsequently incubated with the primary antibodies, including anti-BMP6 antibody (cat no. ab155963; 1:200; Abcam) and anti-VEGF antibody (cat no. ab81289; 1:200; Abcam) overnight at $4^{\circ} \mathrm{C}$, followed by biotinylated goat anti-mouse immunoglobulin G secondary antibodies (cat no. BA1300; 1:500, BD Biosciences) and treated with streptavidin-horseradish peroxidase (BD Biosciences). Tissues were then treated with 

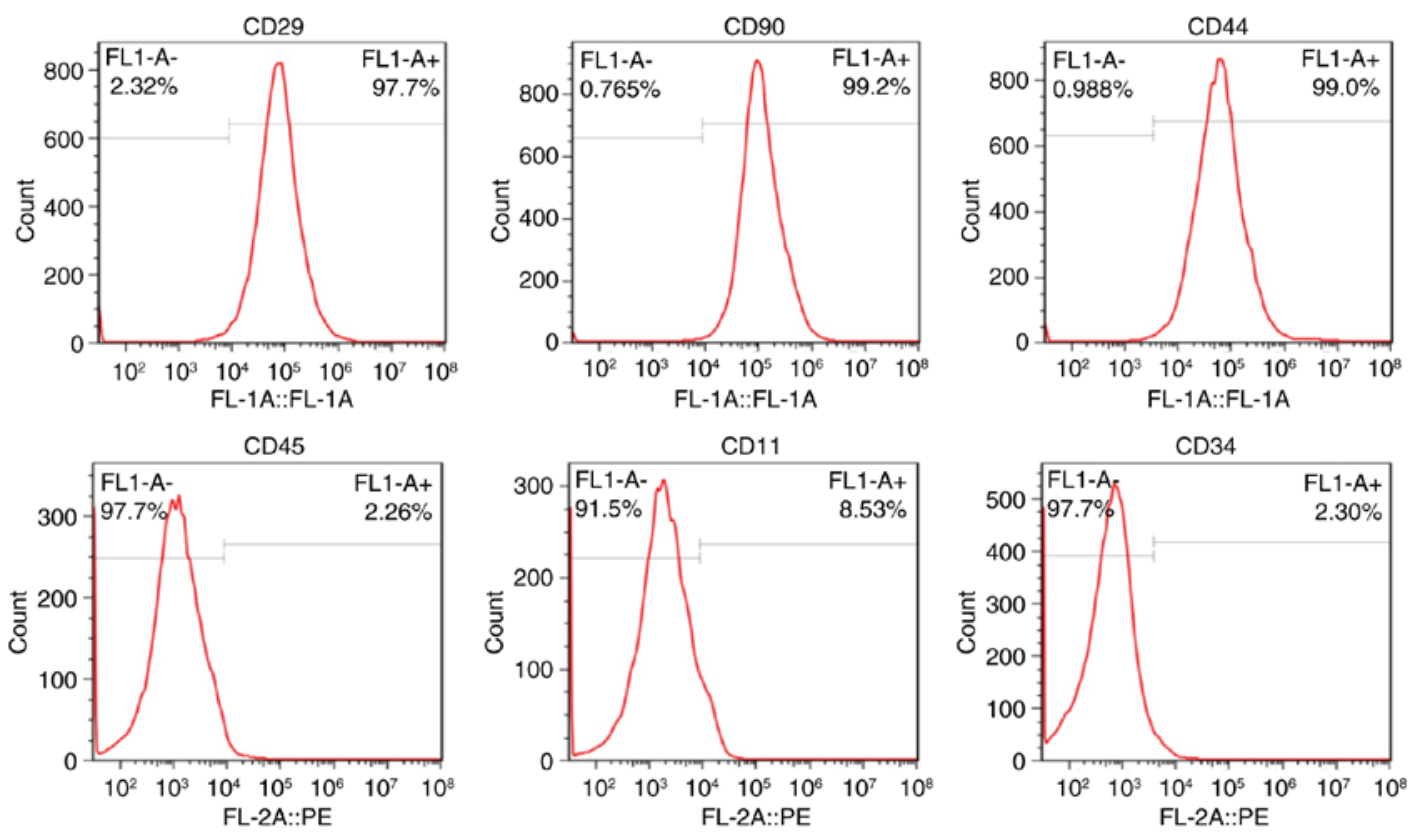

Figure 1. Representative expression of BMSC surface markers CD29, CD90, CD44, CD45, CD11 and CD34 as analyzed by flow cytometry. BMSCs were observed to hyperexpress CD29 (97.7\%), CD90 (99.2\%) and CD44 (99.0\%), whereas CD45 (2.26\%), CD11 (8.53\%) and CD34 (2.30\%) were hypoexpressed. BMSC, bone mesenchymal stem cell; CD, cluster of differentiation; FL-1, fluorescence-1.

3,3'-diaminobenzidine substrate and hematoxylin for $15 \mathrm{~min}$ at room temperature. The data was detected using Image J software 4.8 (National Institutes of Health, Bethesda, MA, USA).

Analysis of blood vessel formation. Formalin-fixed paraffin-embedded tissue sections were immunostained for smooth muscle $\alpha$-actin in order to detect blood vessel formation. Following washing in PBS containing 0.1\% (wt/vol) saponin and 2\% (wt/vol) bovine serum albumin (Gibco; Thermo Fisher Scientific, Inc.), CD34 was quantified by immunohistochemistry in order to detect the number of capillaries. The sections were labeled for CD34 with CY3-conjugated monoclonal anti-CD34 antibody (cat no ab81289; 1:200; Abcam) and incubated overnight at $4^{\circ} \mathrm{C}$. Images of tissue sections were subsequently acquired under a light microscope (magnification, x200) and captured using an Olympus MicroFire color digital camera and PictureFrame image acquisition software 2.0 (Optronics, Goleta, CA, USA). Histological sections were then examined to quantify the blood vessel density on the scaffolds (four sections per sample). Blood vessels were manually counted on the total scaffold and a circular luminal structure was taken to indicate a blood vessel.

Statistical analysis. Data were analyzed with the Student's t-test and one way analysis of variance followed by a Tukey's post hoc test using SPSS 19.0 software (IBM Corp., Armonk, NY, USA). Each experiment was repeated at least three times. All results were summarized and are presented as means \pm standard deviation. $\mathrm{P}<0.05$ was considered to indicate a statistically significant difference.

\section{Results}

Cell verification. The expression of cell surface antigens CD29, CD90, CD44, CD45, CD11 and CD34 were 97.7, 99.2, 99.0,
2.26, 8.53 and $2.3 \%$ positive, respectively (Fig. 1). The cells were demonstrated to high-express CD29, CD90 and CD44, whereas CD45, CD11 and CD34 were low-expressed, indicating that the cells were BMSCs rather than hematopoietic cells.

Expression of VEGF and BMP-6. The relative mRNA expression of BMP-6, VEGF, OCN, RUNX2 and AKP was quantified in the BMSCs 6 weeks post-transfection. VEGF mRNA expression in the AAV-VEGF-BMP-6 and AAV-VEGF groups was significantly higher compared with the AAV-GFP group $(\mathrm{P}<0.05$, Fig. 2A). In addition, BMP-6 mRNA expression in the AAV-VEGF-BMP-6 $(\mathrm{P}<0.01)$ and AAV-BMP-6 $(\mathrm{P}<0.05)$ groups were significantly higher compared with the AAV-GFP group (Fig. 2A). The expression levels of OCN, RUNX2 and AKP mRNA were all upregulated in the AAV-BMP-6 and AAV-VEGF-BMP-6 groups compared with the AAV-GFP group ( $\mathrm{P}<0.05,{ }^{* *} \mathrm{P}<0.01$, Fig. $2 \mathrm{~A}$ ); Western blotting revealed a similar pattern for VEGF and BMP-6 protein expression levels, The expression of BMP6 protein was significantly increased in the AAV-VEGF-BMP-6 group $(\mathrm{P}<0.01)$ and AAV-BMP-6 group $(\mathrm{P}<0.05)$ when compared with the AAV-GFP group Fig. 2B and $\mathrm{C}$ ). In addition, VEGF expression was significantly higher in the AAV-VEGF-BMP-6 and AAV-VEGF groups compared with the AAV-GFP group (both $\mathrm{P}<0.05$, Fig. 2B and C). ELISA was performed to confirm VEGF and BMP-6 expression. The results revealed that VEGF and BMP-6 were expressed in all groups. And BMP6 production increased in the AAV-BMP6 and AAV-VEGF-BMP-6 groups compared with the AAV-GFP group $(\mathrm{P}<0.05, \mathrm{P}<0.01$, Fig. 2D); however, BMP6 production in the AAV-VEGF-BMP6 group was marked greater than that in AAV-BMP-6 group ( $\mathrm{P}<0.05$, Fig. 2D), which suggests that VEGF-BMP6 co-expression promotes the secretion of BMP6. Similarly, VEGF production in the AAV-VEGF and AAV-VEGF-BMP6 groups was significantly increased compared with the AAV-GFP group (Fig. 2E). 

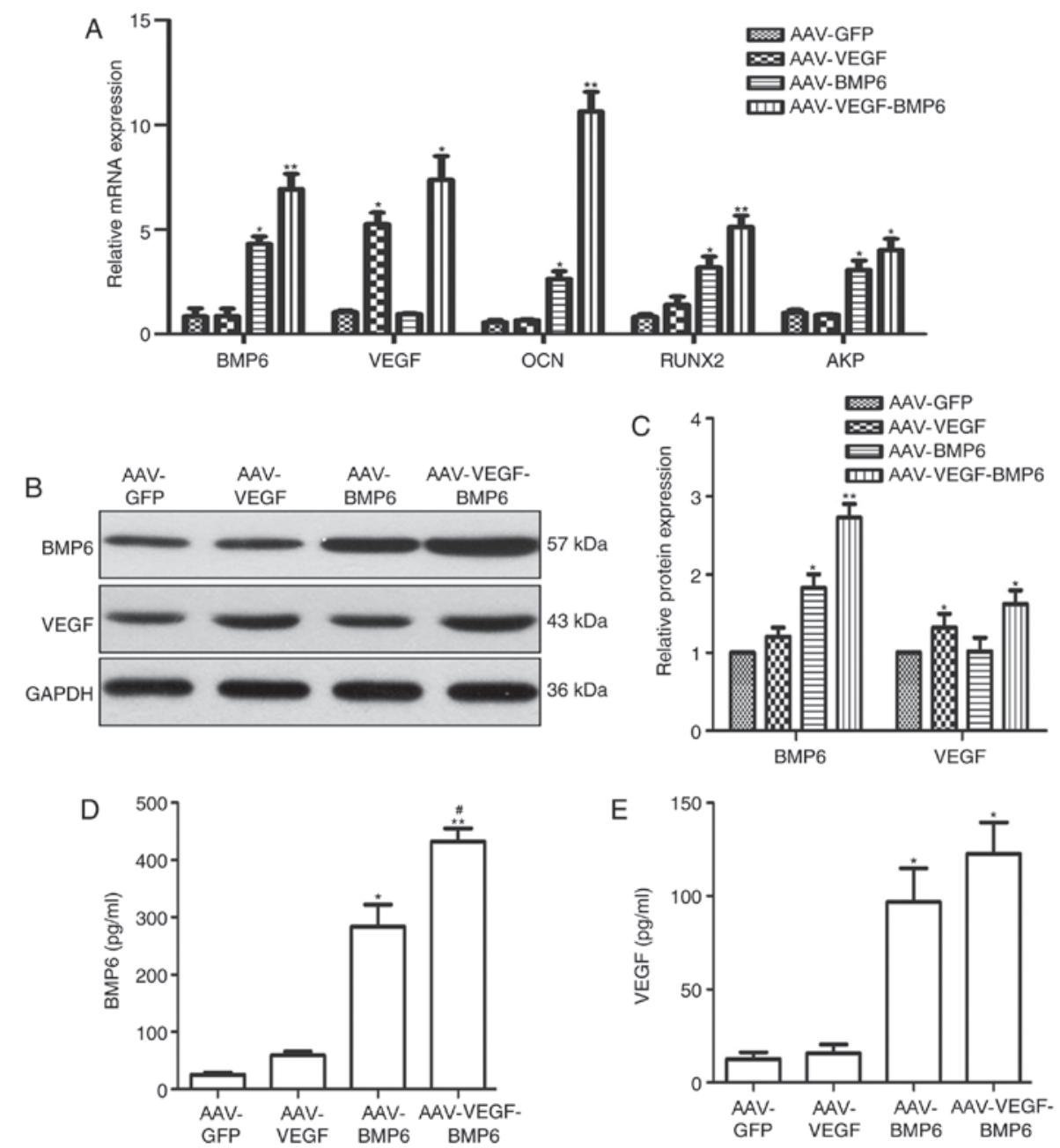

Figure 2. Levels of VEGF and BMP-6 in the AAV-GFP, AAV-BMP-6, AAV-VEGF and AAV-VEGF-BMP groups. (A) Relative mRNA expressions of BMP-6, VEGF, OCN, RUNX2 and AKP analyzed by reverse transcription-quantitative polymerase chain reaction. Levels of VEGF and BMP-6 protein in transfected BMSCs were (B) assessed using western blotting and (C) quantified using densitometry analysis. GAPDH was used as an internal control. ELISA kits were used to measure (D) BMP-6 and (E) VEGF protein expression. "P<0.05 vs. AAV-GFP group, ${ }^{* *} \mathrm{P}<0.01$ vs. AAV-GFP group, ${ }^{*} \mathrm{P}<0.05$ vs. AAV-BMP-6 group. VEGF, vascular endothelial growth factor; BMP, bone morphogenetic protein; AAV, adeno-associated virus; GFP, green fluorescent protein; OCN, osteocalcin; RUNX2, runt-related transcription factor 2; AKP, alkaline phosphatase; BMSCs, bone mesenchymal stem cells.

Biological activity of VEGF and BMP6 in vitro. VEGF secreted from BMSCs in the AAV-VEGF-BMP-6 group enhanced HUVEC proliferation and tube formation in comparison with the other groups (Fig. 3A). Tube formation was significantly increased in the AAV-VEGF-BMP-6 and AAV-VEGF groups compared with the AAV-GFP group $(\mathrm{P}<0.05, \mathrm{P}<0.01$, Fig. 3B). The mineralization effects of BMP-6 were detected using alizarin red, AKP and Von Kossa staining (Fig. 4). The intensity of staining was markedly increased in the AAV-VEGF-BMP-6 group compared with all other groups, these results indicated that co-expressing the VEGF and BMP-6 in BMSCs could enhanced HUVEC tube formation and increased osteogenic ability.

Histological assessment. Histological analysis with H\&E and Von Kossa staining was used to detect bone vessel and bone formation in vivo. H\&E staining analysis revealed that maximum bone formation was achieved in PLAGA implants with BMSCs transfected with VEGF and BMP-6. The greatest number of tubes and strongest capillary integrity were observed in BMSCs transfected with VEGF and BMP-6, with a significant increase in capillaries compared with the
AAV-GFP group ( $\mathrm{P}<0.05$; Fig. 5). The expression of VEGF and BMP-6 in PLAGA implants was examined and it was observed that VEGF and BMP-6 expression was markedly increased in the implants that carried BMSC cells expressing VEGF and BMP-6 compared with the other groups (Fig. 6). Histological analysis with Von Kossa staining was consistent with the H\&E staining results. Furthermore, cellularity and mineral deposition in the AAV-VEGF-BMP-6 implants was markedly increased compared with all other groups (Fig. 6). In order to further assess the effect of VEGF delivery on angiogenesis, immunostaining for CD34 was used to detect the number of capillaries. The results were consistent in indicating that scaffolds containing BMSCs transfected with VEGF and BMP- 6 generated the largest number of blood vessels.

\section{Discussion}

With the rapid development of gene therapy, it is being increasingly recognized as a potential novel therapeutic option for the treatment of ANFH (2). Neovascularization and bone 
A

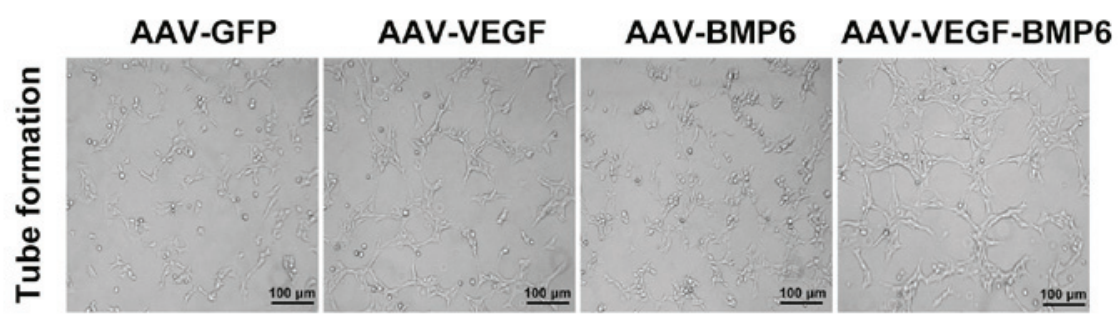

B

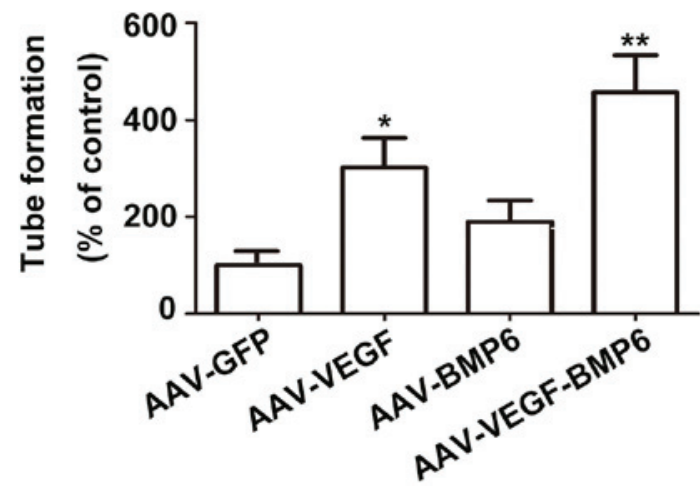

Figure 3. VEGF/BMP-6 stimulated angiogenesis as determined by tube formation of HUVECs in vitro. (A) Representative images (magnification, x200; Scale bar, $100 \mu \mathrm{m}$ ) and (B) quantification of tube formation. Scale bar, $100 \mu \mathrm{m}$. ${ }^{*} \mathrm{P}<0.05$ vs. AAV-GFP group and ${ }^{* *} \mathrm{P}<0.01$ vs. AAV-GFP group. AAV, adeno-associated virus; GFP, green fluorescent protein; VEGF, vascular endothelial growth factor; BMP, bone morphogenetic protein.

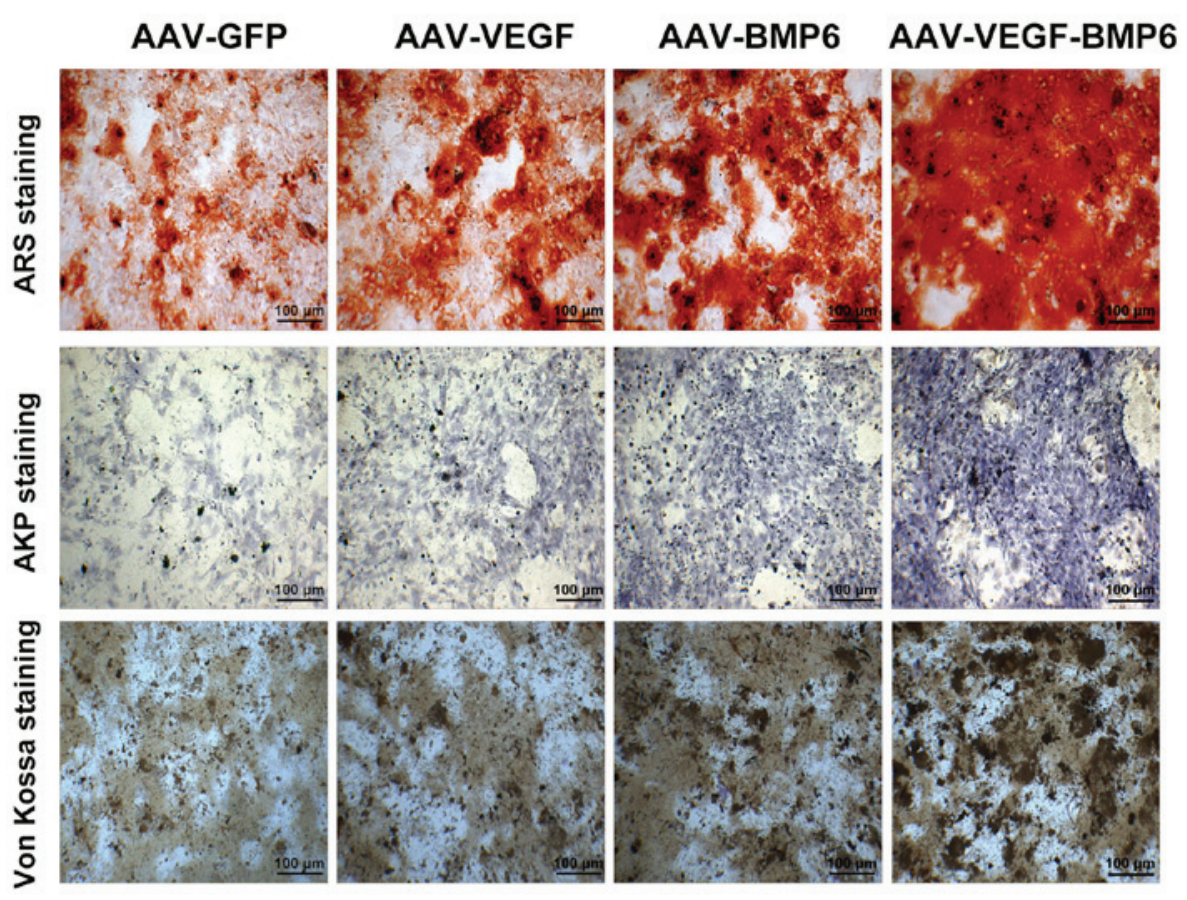

Figure 4. The mineralization effects of VEGF and BMP-6 on BMSCs. Representative images of ARS staining, AKP staining, and Von Kossa staining in the four groups. The mineralization of ARS staining is seen as mineralized nodules. The mineralization of BMSCs stained using the AKP and Von Kossa methods is represented by black dots. Scale bar, $100 \mu \mathrm{m}$. ARS, alizarin red staining; AKP, alkaline phosphatase; AAV, adeno-associated virus; GFP, green fluorescent protein; VEGF, vascular endothelial growth factor; BMP, bone morphogenetic protein; BMSCs, bone mesenchymal stem cells.

formation through the functions of VEGF and BMP have been extensively studied (32). The AAV vector is an attractive non-pathogenic human viral vector with low immunogenicity (33). It is efficient for gene transduction in vitro and for local injection in vivo in the presence of a helper virus, for example adenovirus or herpes virus $(34,35)$. In the present study, therefore, AAV was used to transfer and correctly express the target gene.

Angiogenesis and osteogenesis are critical for bone renovation and are associated with a number of cytokines, including 
A

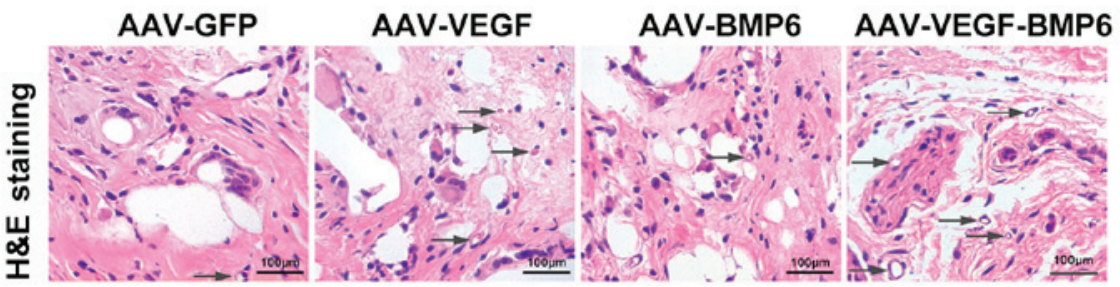

B

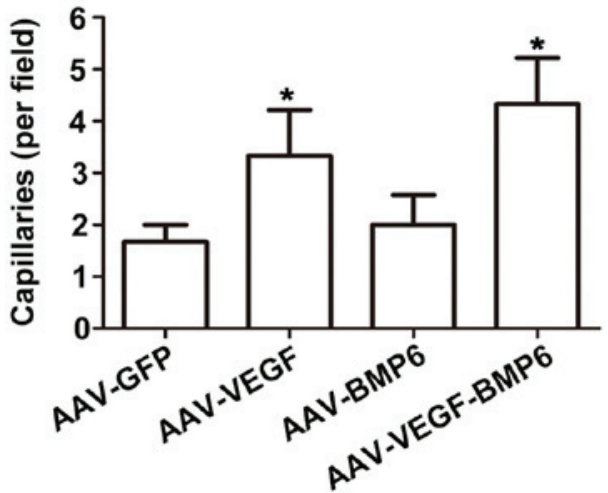

Figure 5. H\&E staining of BMSCs implanted into PLAGA. (A) H\&E staining of AAV-GFP, AAV-BMP-6, AAV-VEGF and AAV-VEGF-BMP-6 groups was performed to detect in vivo blood vessel formation. Blood vessels were identified by the presence of a luminal structure, and the arrows point to the blood vessels. Scale bar, $100 \mu \mathrm{m}$. (B) The number of capillaries in the four groups was quantified. "P<0.05 vs. AAV-GFP group. PLAGA, poly lactide-co-glycolide; $\mathrm{H} \& \mathrm{E}$, hematoxylin and eosin; AAV, adeno-associated virus; GFP, green fluorescent protein; VEGF, vascular endothelial growth factor; BMP, bone morphogenetic protein.

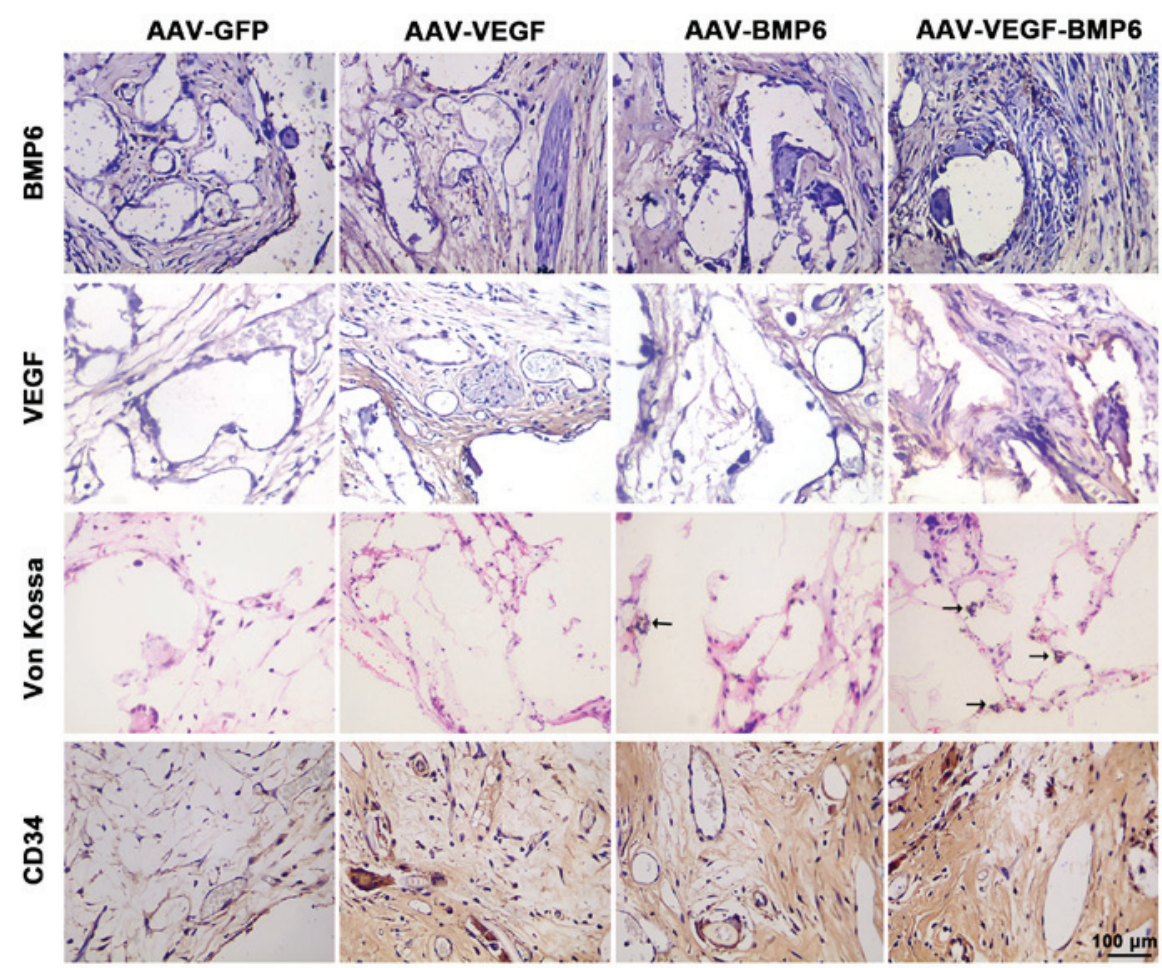

Figure 6. Immunohistochemical staining analysis was used to detect the expression of VEGF, BMP-6 mineral deposition and angiogenesis in PLAGA implants. CD34 was quantified, in order to detect the number of capillaries. Scale bar, $100 \mu \mathrm{m}$. PLAGA, poly lactide-co-glycolide; aav, adeno-associated virus; GFP, green fluorescent protein; VEGF, vascular endothelial growth factor; BMP, bone morphogenetic protein; CD34, cluster of differentiation 34.

bFGF, TGF- $\beta$, BMPs, and VEGF $(4,36)$. The effects of VEGF and BMPs on the process of bone formation have already been extensively studied (37,38). VEGF is important in angiogenesis and is able to induce angiopoiesis by promoting the division and growth of vascular endothelial cells $(7,39)$. In addition to its effects in angiogenesis, several studies have reported 
that VEGF also serves an important role in osteogenesis by interacting with BMPs $(40,41)$. It was therefore hypothesized that VEGF may exhibit distinct effects when combined with different BMPs. Several studies have shown that the VEGF and BMP-7 proteins expressed by the rAAV-human (h)VEGF-IRES-hBMP-7 vector enhanced angiogenesis and bone regeneration both in vitro and in vivo $(30,42)$. In addition, recent studies have reported that BMP-6 and BMP-9 exhibited the most potent osteogenic activity $(43,44)$, and that BMP-6 encourages the differentiation of human stromal cells to osteoblasts (45). Consequently, the combined effects of VEGF and BMP-6 were assessed in the present study. Angiogenic and osteogenic assays were used to identify the biological effects of VEGF and BMP-6 in vitro and in vivo.

The present study demonstrated that the expression of VEGF and BMP-6 was increased in the AAV-VEGF-BMP-6 group in vitro compared with all other groups. VEGF secreted by BMSCs in the AAV-VEGF-BMP-6 group enhanced HUVEC tube formation. Similarly, the osteogenic ability of BMP-6 in the AAV-VEGF-BMP-6 group was significantly higher compared with the other three groups, as demonstrated by alizarin red, AKP and von Kossa staining. These results indicate that the vector co-expressing VEGF and BMP-6 enhanced bone repair and regeneration in vitro. The results in this study are in accord with those of a previous study (46).

BMSCs are a type of multipotent mesenchymal stem cell found in bone marrow (47). They have a high capacity for self-renewal and the potential to differentiate into several cell types, and so have long been considered a source of cells for bone tissue engineering (47). Surface antigen detection using flow cytometry is the gold standard for cell verification; in the present study, CD29, CD90, CD44, CD45, CD11 and CD34 were selected as target cell surface antigens based on previous literature $(48,49)$. The results revealed the hyperexpression of CD29, CD90 and CD44, as well as hypoexpression of CD45, CD11 and CD34, suggesting a high BMSC purity. PLAGA is a biomedical polymer that has been safely used as an implant material for several decades, including in bone tissue development (50). Prior to transplantation, PLAGA was fabricated into a highly porous 3D scaffold upon which BMSCs could be seeded with high efficiency (51). According to Roedersheimer et al (36) and Cui et al (46), VEGF and BMP-6 interact directly at the molecular level or act via their signaling pathways in order to promote osteogenesis.

In the present study, it was hypothesized that VEGF and BMP-6 would act in an additive manner to promote the osteoblastic differentiation of BMSCs in vitro and bone formation in vivo. A composite bone graft substitute containing BMSCs transfected with VEGF, BMP-6 or VEGF/BMP-6 loaded on a PLAGA scaffold was used. The results revealed that there was a significant increase in the bone volume of implants that carried BMSCs expressing both VEGF and BMP-6 compared to those expressing either of the growth factors alone at 2 and 3 weeks following implantation. A greater number of blood vessels were also observed in the AAV-VEGF-BMP-6 group compared with other BMSC groups expressing either growth factor alone. Studies have revealed that VEGF serves as a chemoattractant for mesenchymal stem cells to increase the efficiency of bone formation in the presence of BMP-6 $(52,53)$. As demonstrated in the present study, VEGF and BMP-6 interact directly at the molecular level or via their signaling pathways to indirectly enhance bone formation by increasing vascular formation. The increased bone growth and angiogenesis in subcutaneously implanted PLAGA scaffolds was in accordance with the in vitro results.

In conclusion, co-expression of VEGF and BMP-6 in BMSCs enhanced angiogenesis and bone regeneration in vitro and in vivo. This demonstrates that BMSCs expressing both VEGF and BMP-6 are responsible for increased numbers of blood vessels and increased bone formation, which provides theoretical support for their use in ANFH gene therapy.

\section{Acknowledgements}

The present study was supported by the Natural Science Foundation of Guangdong Province, China (grant no. 2014A030307006), the National Natural Science Foundation of China (grant no. 31430030) and the Medical Scientific Research Foundation of Guangdong Province, China (grant no. A2017306).

\section{References}

1. Chamberlain JR,Schwarze U,Wang PR,Hirata RK, Hankenson KD, Pace JM, Underwood RA, Song KM, Sussman M, Byers PH and Russell DW: Gene targeting in stem cells from individuals with osteogenesis imperfecta. Science 303: 1198-1201, 2004.

2. Yang C, Yang S, Du J, Li J, Xu W and Xiong Y: Experimental study of vascular endothelial growth factor gene therapy for avascular necrosis of the femoral head. J Huazhong Univ Sci Technolog Med Sci 23: 297-299, 316, 2003.

3. Wilson CG, Martin-Saavedra FM, Vilaboa N and Franceschi RT Advanced BMP gene therapies for temporal and spatial control of bone regeneration. J Dent Res 92: 409-417, 2013.

4. Kuh SU, Zhu Y, Li J, Tsai KJ, Fei Q, Hutton WC and Yoon ST: Can TGF-betal and rhBMP-2 act in synergy to transform bone marrow stem cells to discogenic-type cells? Acta Neurochir (Wien) 150: 1073-1079, 2008

5. Evans $\mathrm{CH}$ and Huard J: Gene therapy approaches to regenerating the musculoskeletal system. Nat Rev Rheumatol 11: 234-242, 2015.

6. Herberg S, Susin C, Pelaez M, Howie RN, Moreno de Freitas R, Lee J, Cray JJ Jr, Johnson MH, Elsalanty ME, Hamrick MW, et al: Low-dose bone morphogenetic protein-2/stromal cell-derived factor- $1 \beta$ cotherapy induces bone regeneration in critical-size rat calvarial defects. Tissue Eng Part A 20: 1444-1453, 2014.

7. White AP, Vaccaro AR, Hall JA, Whang PG, Friel BC and McKee MD: Clinical applications of BMP-7/OP-1 in fractures, nonunions and spinal fusion. Int Orthop 31: 735-741, 2007.

8. La WG, Jin M, Park S, Yoon HH, Jeong GJ, Bhang SH, Park H, Char K and Kim BS: Delivery of bone morphogenetic protein-2 and substance $\mathrm{P}$ using graphene oxide for bone regeneration. Int J Nanomed 9 (Suppl 1): S107-S116, 2014.

9. Wang YJ, Chen S, Deng C, Li F, Wang Y,Hu X, Shi F and Dong N: MicroRNA-204 Targets Runx2 to Attenuate BMP-2-induced osteoblast differentiation of human aortic valve interstitial cells. J Cardiovasc Pharmacol 66: 63-71, 2015.

10. Mundy C, Gannon M and Popoff SN: Connective tissue growth factor (CTGF/CCN2) negatively regulates BMP-2 induced osteoblast differentiation and signaling. J Cell Physiol 229: 672-681, 2014.

11. Hanada K, Solchaga LA, Caplan AI, Hering TM, Goldberg VM, Yoo JU and Johnstone B: BMP-2 induction and TGF-beta 1 modulation of rat periosteal cell chondrogenesis. J Cell Biochem 81: 284-294, 2001.

12. Wang L, Park P, La Marca F, Than KD and Lin CY: BMP-2 inhibits tumor-initiating ability in human renal cancer stem cells and induces bone formation. J Cancer Res Clin Oncol 141: 1013-1024, 2015

13. Dohin B, Dahan-Oliel N, Fassier F and Hamdy R: Enhancement of difficult nonunion in children with osteogenic protein-1 (OP-1): Early experience. Clin Orthop Relat Res 467: 3230-3238, 2009.

14. Takahashi T, Hatakeyama S and Machida T: Ductal adenocarcinoma of the pancreas with psammomatous calcification: Report of a case with immunohistochemical study for bone morphogenetic protein. Pathol Int 61: 603-607, 2011. 
15. Rahman MS, Akhtar N, Jamil HM, Banik RS and Asaduzzaman SM: TGF- $\beta$ /BMP signaling and other molecular events: Regulation of osteoblastogenesis and bone formation. Bone Res 3: 15005, 2015.

16. Scharpfenecker M, van Dinther M, Liu Z, van Bezooijen RL, Zhao Q, Pukac L, Löwik CW and Ten Dijke P: BMP-9 signals via ALK1 and inhibits bFGF-induced endothelial cell proliferation and VEGF-stimulated angiogenesis. J Cell Sci 120: 964-972, 2007.

17. Simic P, Culej JB, Orlic I, Grgurevic L, Draca N, Spaventi R and Vukicevic S: Systemically administered bone morphogenetic protein- 6 restores bone in aged ovariectomized rats by increasing bone formation and suppressing bone resorption. J Biol Chem 281: 25509-25521, 2006.

18. Mizrahi O, Sheyn D, Tawackoli W, Kallai I, Oh A, Su S, Da X, Zarrini P, Cook-Wiens G, Gazit D and Gazit Z: BMP-6 is more efficient in bone formation than BMP-2 when overexpressed in mesenchymal stem cells. Gene Ther 20: 370-377, 2013.

19. Street J, Bao M, deGuzman L, Bunting S, Peale FV Jr, Ferrara N, Steinmetz H, Hoeffel J, Cleland JL, Daugherty A, et al: Vascular endothelial growth factor stimulates bone repair by promoting angiogenesis and bone turnover. Proc Natl Acad Sci USA 99: 9656-9661, 2002

20. Moens S, Goveia J, Stapor PC, Cantelmo AR and Carmeliet P The multifaceted activity of VEGF in angiogenesis-Implications for therapy responses. Cytokine Growth Factor Rev 25: 473-482, 2014.

21. Gerber HP, Vu TH, Ryan AM, Kowalski J, Werb Z and Ferrara N: VEGF couples hypertrophic cartilage remodeling, ossification and angiogenesis during endochondral bone formation. Nat Med 5: 623-628, 1999.

22. Ferrara N: VEGF-A: A critical regulator of blood vessel growth. Eur Cytokine Netw 20: 158-163, 2009.

23. Joensuu K, Uusitalo L, Alm JJ, Aro HT, Hentunen TA and Heino TJ: Enhanced osteoblastic differentiation and bone formation in co-culture of human bone marrow mesenchymal stromal cells and peripheral blood mononuclear cells with exogenous VEGF. Orthop Traumatol Surg Res 101: 381-386, 2015.

24. Ramazanoglu M, Lutz R, Rusche P, Trabzon L, Kose GT, Prechtl C and Schlegel KA: Bone response to biomimetic implants delivering BMP-2 and VEGF: An immunohistochemical study. J Craniomaxillofac Surg 41: 826-835, 2013.

25. Zhang W, Zhu C, Wu Y, Ye D, Wang S, Zou D, Zhang X, Kaplan DL and Jiang X: VEGF and BMP-2 promote bone regeneration by facilitating bone marrow stem cell homing and differentiation. Eur Cells Mater 27: 1-12, 2014

26. Kempen DH, Lu L, Heijink A, Hefferan TE, Creemers LB, Maran A, Yaszemski MJ and Dhert WJ: Effect of local sequential VEGF and BMP-2 delivery on ectopic and orthotopic bone regeneration. Biomaterials 30: 2816-2825, 2009.

27. Peng H, Usas A, Olshanski A, Ho AM, Gearhart B, Cooper GM and Huard J: VEGF improves, whereas sFlt1 inhibits, BMP2-induced bone formation and bone healing through modulation of angiogenesis. J Bone Miner Res 20: 2017-2027, 2005.

28. Pittenger MF, Mackay AM, Beck SC, Jaiswal RK, Douglas R, Mosca JD, Moorman MA, Simonetti DW, Craig S and Marshak DR: Multilineage potential of adult human mesenchymal stem cells. Science 284: 143-147, 1999.

29. Büning $\mathrm{H}$, Perabo L, Coutelle O, Quadt-Humme S and Hallek M: Recent developments in adeno-associated virus vector technology. J Gene Med 10: 717-733, 2008.

30. Shi ZB and Wang KZ: Effects of recombinant adeno-associated viral vectors on angiopoiesis and osteogenesis in cultured rabbit bone marrow stem cells via co-expressing hVEGF and hBMP genes: A preliminary study in vitro. Tissue Cell 42: 314-321, 2010.

31. Livak KJ and Schmittgen TD: Analysis of relative gene expression data using real-time quantitative PCR and the 2(-Delta Delta C(T)) method. Methods 25: 402-408, 2012.

32. Xin CW, Zheng LW, Lei LC, Ma L, Ehrbar M, Weber FE and Zwahlen RA: Effect of different rhBMP-2 and TG-VEGF ratios on the formation of heterotopic bone and neovessels. Biomed Res Int 2014: 571510, 2014.

33. Ali RR, Reichel MB, Thrasher AJ, Levinsky RJ, Kinnon C, Kanuga N, Hunt DM and Bhattacharya SS: Gene transfer into the mouse retina mediated by an adeno-associated viral vector. Hum Mol Genet 5: 591-594, 1996.

34. Merten OW, Gény-Fiamma C and Douar AM: Current issues in adeno-associated viral vector production. Gene Ther 12 (Suppl 1): S51-S61, 2005.
35. Grieger JC, Choi VW and Samulski RJ: Production and characterization of adeno-associated viral vectors. Nat Protoc 1: 1412-1428, 2006.

36. Roedersheimer M, West J, Huffer W, Harral J and Benedict J: A bone-derived mixture of TGF beta-superfamily members forms a more mature vascular network than bFGF or TGF-beta 2 in vivo. Angiogenesis 8: 327-338, 2005.

37. Samee M, Kasugai S, Kondo H, Ohya K, Shimokawa H and Kuroda S: Bone morphogenetic protein-2 (BMP-2) and vascular endothelial growth factor (VEGF) transfection to human periosteal cells enhances osteoblast differentiation and bone formation. J Pharmacol Sci 108: 18-31, 2008.

38. Sukul M, Nguyen TB, Min YK, Lee SY and Lee BT: Effect of local sustainable release of BMP2-VEGF from Nano-cellulose loaded in sponge biphasic calcium phosphate on bone regeneration. Tissue Eng Part A 21: 1822-1836, 2015.

39. Cao QS, Zhang T and Zhang J: Correlation analysis of STAT3 and VEGF expression and eosinophil infiltration in nasal polyps. Eur Arch Otorhinolaryngol 272: 1955-1960, 2015.

40. Kanczler JM, Ginty PJ, White L, Clarke NM, Howdle SM, Shakesheff KM and Oreffo RO: The effect of the delivery of vascular endothelial growth factor and bone morphogenic protein-2 to osteoprogenitor cell populations on bone formation. Biomaterials 31: 1242-1250, 2010.

41. Jiang J, Wang SH, Chai F, Ai CC and Chen SY: The study on vascularisation and osteogenesis of BMP/VEGF co-modified tissue engineering bone in vivo. RSC Adv 6: 41800-41808, 2016.

42. Zhang C, Wang KZ, Qiang H, Tang YL, Li Q, Li M and Dang XQ: Angiopoiesis and bone regeneration via co-expression of the hVEGF and hBMP genes from an adeno-associated viral vector in vitro and in vivo. Acta Pharmacol Sin 31: 821-830, 2010.

43. Yung LM, Sánchez-Duffhues G, Ten Dijke P and Yu PB: Bone morphogenetic protein 6 and oxidized Low-density lipoprotein synergistically recruit osteogenic differentiation in endothelial cells. Cardiovasc Res 108: 278-287, 2015.

44. Wei Z, Salmon RM, Upton PD, Morrell NW and Li W: Regulation of bone morphogenetic protein 9 (BMP9) by redox-dependent proteolysis. J Biol Chem 289: 31150-31159, 2014.

45. Friedman MS, Long MW and Hankenson KD: Osteogenic differentiation of human mesenchymal stem cells is regulated by bone morphogenetic protein-6. J Cell Biochem 98: 538-554, 2006.

46. Cui F, Wang X, Liu X, Dighe AS, Balian G and Cui Q: VEGF and BMP- 6 enhance bone formation mediated by cloned mouse osteoprogenitor cells. Growth Factors 28: 306-317, 2010

47. Derubeis AR and Cancedda R: Bone marrow stromal cells (BMSCs) in bone engineering: Limitations and recent advances. Ann Biomed Eng 32: 160-165, 2004.

48. Xiong J, Menicanin D, Zilm PS, Marino V, Bartold PM and Gronthos S: Investigation of the cell surface proteome of human periodontal ligament stem cells. Stem Cells Int 2016: 1947157, 2016.

49. Hung SC, Chen NJ, Hsieh SL, Li H, Ma HL and Lo WH: Isolation and characterization of size-sieved stem cells from human bone marrow. Stem Cells 20: 249-258, 2002.

50. Bongio M, van den Beucken JJJP, Leeuwenburgh SCG and Jansen JA: Development of bone substitute materials: From 'biocompatible' to 'instructive'. J Mater Chem 20: 8747-8759, 2010.

51. Nugroho RWN, Odelius K, Hoglund A and Albertsson AC: Highlighting the importance of surface grafting in combination with a Layer-by-Layer approach for fabricating advanced 3D Poly(L-lactide) microsphere scaffolds. Chem Mater 28: 3298-3307, 2016.

52. Madhu V, Li CJ, Dighe AS, Balian G and Cui Q BMP-non-responsive Sca $1^{+} \mathrm{CD} 73^{+} \mathrm{CD} 44^{+}$mouse bone marrow derived osteoprogenitor cells respond to combination of VEGF and BMP- 6 to display enhanced osteoblastic differentiation and ectopic bone formation. PLoS One 9: e103060, 2014.

53. Fiedler J, Leucht F, Waltenberger J, Dehio C and Brenner RE: VEGF-A and PIGF-1 stimulate chemotactic migration of human mesenchymal progenitor cells. Biochem Biophys Res Commun 334: 561-568, 2005 .

This work is licensed under a Creative Common Attribution-NonCommercial-NoDerivatives 4.0 International (CC BY-NC-ND 4.0) License. 